\title{
Comparison of the Effects of Nonlinear Gain and Weak Optical Feedback on the Dynamics of Semiconductor Lasers
}

\author{
C. Masoller
}

\begin{abstract}
The influence of nonlinear gain and optical feedback on the dynamics of single-mode semiconductor lasers are numerically investigated based on the Lang and Kobayashi model. It is well known that the nonlinear gain tends to stabilize the dynamics, while the optical feedback tends to increase the instabilities. In this paper, we study the behavior of the attractors when the feedback level $k$ and the gain saturation coefficient $\varepsilon$ vary and show that the effects of these parameters are surprisingly opposite. For example, we find that the route to chaos that the external cavity modes follow for increasing $k$ is reversed for increasing $\varepsilon$ in an almost identical manner. When the feedback increases the modes follow the usual quasi-periodic route and turn into torus. If $k$ continues to increase, the torus become chaotic attractors as the result of several period-doubling bifurcations or a third Hopf bifurcation. Further increase of $k$ causes the chaotic attractors to lose stability. Contrarily, if the value of the parameter $\varepsilon$ is increased, the attractors recover their stability and reverse the route becoming simple torus again. If $\varepsilon$ is increased further, the torus reverse the quasi-periodic route and turn into stable modes again. We also find that on the contrary to $k$, the parameter $\varepsilon$ enhances the stability region of an attractor. We show that the feedback level above which a limit cycle emerges from a stable mode, the feedback level above which a torus emerges from a limit cycle, and the feedback level above which a chaotic attractor loses stability are all increasing functions of $\varepsilon$.
\end{abstract}

\section{INTRODUCTION}

$\mathbf{T}$ HE INTENSITY reduction of the gain is a phenomenon that has attracted considerable attention recently due to its strong influence in semiconductor laser dynamics. It is usually referred to as gain saturation or nonlinear gain and affects the damping rate of relaxation oscillations, the modulation response, and the stability and spectral properties of the laser. Although the physical processes that contribute to gain nonlinearities are still not fully understood, it has been shown that spatial and spectral hole burning, carrier heating, and twophoton absorption are phenomena responsible for nonlinear gain [1], [2].

The inclusion of gain saturation in the governing rate equations is known to be important in order to describe accurately the dynamics of semiconductor lasers, and various models have been advanced to account for gain nonlinearities.

Manuscript received June 7, 1996; revised September 4, 1996. This work was supported by Project 47 of the BID-CONICYT Program of the Consejo Nacional de Ciencia y Tecnologia (CONICYT) of Uruguay, the Comision Sectorial de Investigacion Cientifica (CSIC), and the PEDECIBA.

The author is with the Instituto de Física, Facultad de Ciencias, Universidad de la República, Montevideo, Uruguay.

Publisher Item Identifier S 0018-9197(97)03039-X.
When the laser operates in a single longitudinal mode, the saturation is included phenomenologically in the rate equations by writing the modal gain per unit time $G$ with an explicit intensity dependence

$$
G\left(N, E^{2}\right)=G_{N}\left(N-N_{o}\right)\left(1-\varepsilon E^{2}\right)
$$

where $G_{N}$ is the modal gain coefficient, $E$ is the field amplitude, i.e., $E^{2}$ is proportional to the total photon number $I, N$ is the carrier density, $N_{o}$ the carrier density at transparency, and $\varepsilon$ the gain saturation coefficient. The form (1) is valid for low output powers, since for large $\varepsilon E^{2}$ the expression is negative. The alternative forms

$$
\begin{aligned}
& G_{a}\left(N, E^{2}\right)=\frac{G_{N}\left(N-N_{o}\right)}{1+\varepsilon E^{2}} \\
& G_{b}\left(N, E^{2}\right)=\frac{G_{N}\left(N-N_{o}\right)}{\sqrt{1+2 \varepsilon E^{2}}}
\end{aligned}
$$

have been proposed and are commonly employed. For optical intensities much below the saturation level, $G_{a}$ and $G_{b}$ become equivalent to $G$ by expanding to first order in $\varepsilon E^{2}$.

It is well known that semiconductor lasers provide a highly stable radiation field. However, even a small amount of optical feedback from an external reflector might have a profound impact on the dynamic and spectral behavior of the laser. Low levels of optical feedback might be used to obtain a significant linewidth reduction and improved frequency stability, but higher feedback levels might cause the laser to switch to the coherence collapse state, in which the laser linewidth increases to several gigahertz, and the dynamics is chaotic.

The theoretical studies of a semiconductor laser coupled to an external cavity are commonly based on the Lang and Kobayashi model [3], which has proven to successfully describe the observed laser behavior [4], [5]. The model consists of rate equations for the complex electric field $E(t)$ and for the carrier density $N(t)$ inside the laser cavity. The field equation contains a single time-delayed term that takes into account the field fed back into the laser cavity, and therefore, since multiple reflections are neglected, the model is valid for weak feedback levels.

Two of the parameters that have important effects on the response of the laser are the linewidth enhancement factor $\alpha$ [6] and the gain saturation coefficient $\varepsilon$. The destabilizing effect of the former and the stabilizing effect of the latter have been reported in several papers [7]-[9]. In a previous 
work [10], it was shown that the gain saturation plays an important role in the dynamic response of the laser, increasing the feedback level above which the transition to coherence collapse occurs. However, we found that the particular form of nonlinear gain employed in the Lang and Kobayashi model had no important effects on the dynamics, the three forms $G, G_{a}$, and $G_{b}$ were tested and the resulting dynamics was almost independent of the form used, but strongly sensitive to the value of the parameter $\varepsilon$.

In the first part of our present investigation [11] (hereafter called part I), we studied two different sets of Lang and Kobayashi equations for a single-mode semiconductor laser. The two sets differ in the way the $\alpha$ parameter is introduced in the model. The linewidth enhancement factor is introduced in the field equation by linearizing the frequency of the laser mode around its threshold value. One of the studied sets [(5) and (3) of part I)] results from taking into account the intensity reduction of $G$ in the linearization of $\omega$, while the other set [(6) and (3) of part I)] results from neglecting it. As we have shown in part I, the two sets are equal when a linear form for $G$ is assumed, but when gain nonlinearities are taken into account, the behavior they predict is strikingly different. Nevertheless, in both sets, it was found that the parameter tends to stabilize the dynamics, while the feedback level $k$ tends to increase the instabilities.

A general question arising from the previous studies is to what extent the effects of nonlinear gain and weak optical feedback can be considered opposite. In this paper, we focus our attention on comparing the effects of the parameters $k$ and $\varepsilon$ on the dynamics of (5) and (3) of part I. We find several interesting results that demonstrate that the effects of these parameters are surprisingly opposite.

First, we find that if the parameters $k$ and $\varepsilon$ are both increased or decreased certain amounts $(\Delta k, \Delta \varepsilon)$, the Poincaré section of an attractor (which is the transversal cut of the attractor with a two-dimensional plane [12]) does not change significantly. The value of $\Delta \varepsilon$ that compensates the variation $\Delta k$ depends on the attractor, but in all the studied attractors we have found that if $\Delta k>0$, then $\Delta \varepsilon>0$, i.e., an increase of the feedback might be compensated by an increase of the gain saturation, and the dynamics of the laser remains unchanged.

Second, we find that when the value of the parameter $\varepsilon$ is increased the attractors reverse, in an almost identical manner, the route to chaos that they followed for increasing feedback. As it is well established, as $k$ increases the external cavity modes (which are the stationary solutions of the rate equations) follow a quasi-periodic route and become torus. Further increase of $k$ causes a torus to become a chaotic attractor, either after several period-doubling bifurcations or after the apparition of a third incommensurate frequency (i.e., after the occurrence of a third Hopf bifurcation). Contrarely, if the value of the parameter $\varepsilon$ is increased a chaotic attractor reverses the route, becoming a simple torus again. If it underwent several period-doubling bifurcations for increasing $k$, it undergoes several inverse period-doubling bifurcations for increasing $\varepsilon$, or if it underwent a third Hopf bifurcation for increasing $k$, it undergoes an inverse Hopf bifurcation for increasing $\varepsilon$. Moreover, if $\varepsilon$ is increased further, the torus reverses the quasi-periodic route and becomes a stable mode again.

Also, we find that a slight increase of the feedback above a certain critical value causes a chaotic attractor to lose stability, but a slight increase of the value of $\varepsilon$ renders the attractor stable again. The critical feedback level above which an attractor loses stability $\left(k_{c}\right)$ depends on the value of the parameter $\varepsilon$. We studied in detail the behavior of two attractors: attractor A (originated from the perturbed laser mode) and attractor B (originated from the first compound cavity mode). For both attractors, $k_{c}$ is found to increase almost linearly with $\varepsilon$, i.e., the higher the value of $\varepsilon$, the higher the feedback level above which the attractor loses stability.

Furthermore, we find that both the feedback level above which a limit cycle emerges from a stable mode $\left(k_{l c}\right)$ and the feedback level above which a torus emerges from a limit cycle $\left(k_{t}\right)$ also increase with the value of the parameter $\varepsilon$. We show that the numerical value of $k_{l c}$ of a given mode and its dependency with the parameter $\varepsilon$ is in very good agreement with the analytical expression derived from a small-signal analysis of the Lang and Kobayashi model.

Finally, and with the aim of comparing the effects of the parameters $k$ and $\varepsilon$ in the coherence collapse state, we revisited the results of [13], where we studied theoretically and experimentally the visibility (i.e., the field autocorrelation function) of a semiconductor laser operated well above the onset of coherence collapse. In [13], four experimentally measured visibility curves, corresponding to four different attenuations of the light, were numerically fitted calculating the field autocorrelation function based on the Lang and Kobayashi model. Good results were found varying the parameters $k$ and $\varepsilon$; the higher the value of $\varepsilon$ used, the higher the feedback levels that had to be employed in order to fit the visibility measurements. Here, we show that the plots of the feedback levels as a function of $\varepsilon$ give four straight lines with the same positive slope. This indicates that the visibility of the laser within the coherence collapse regime remains approximately unchanged if the parameters $k$ and $\varepsilon$ are varied accordingly to the linear relation.

In summary, the main conclusion of our study is that the effects of the parameters $k$ and $\varepsilon$ are surprisingly opposite, in spite of the fact that they appear in terms of the field rate equations that represent physically independent contributions to the rate of variation of the complex electric field. We have investigated parameter regions where the dynamics is very different, i.e., we have studied the stationary, periodic, and quasi-periodic behavior, the onset of coherence collapse, and well above the onset of coherence collapse, and in all regions we found that mainly the effect of nonlinear gain is to enhance the stability region of an attractor by increasing the feedback rate required to destabilize it.

This paper is organized as follows. Section II presents the result obtained by studying the behavior of the attractors when $k$ and $\varepsilon$ are varied. We also show that the values of the parameters $k$ and $\varepsilon$ used in [13] to fit the visibility curves satisfy linear relations with the same positive slope. In Section III, we present our conclusions. A small-signal analysis of (5) and (3) of part I is presented in the Appendix, and an 

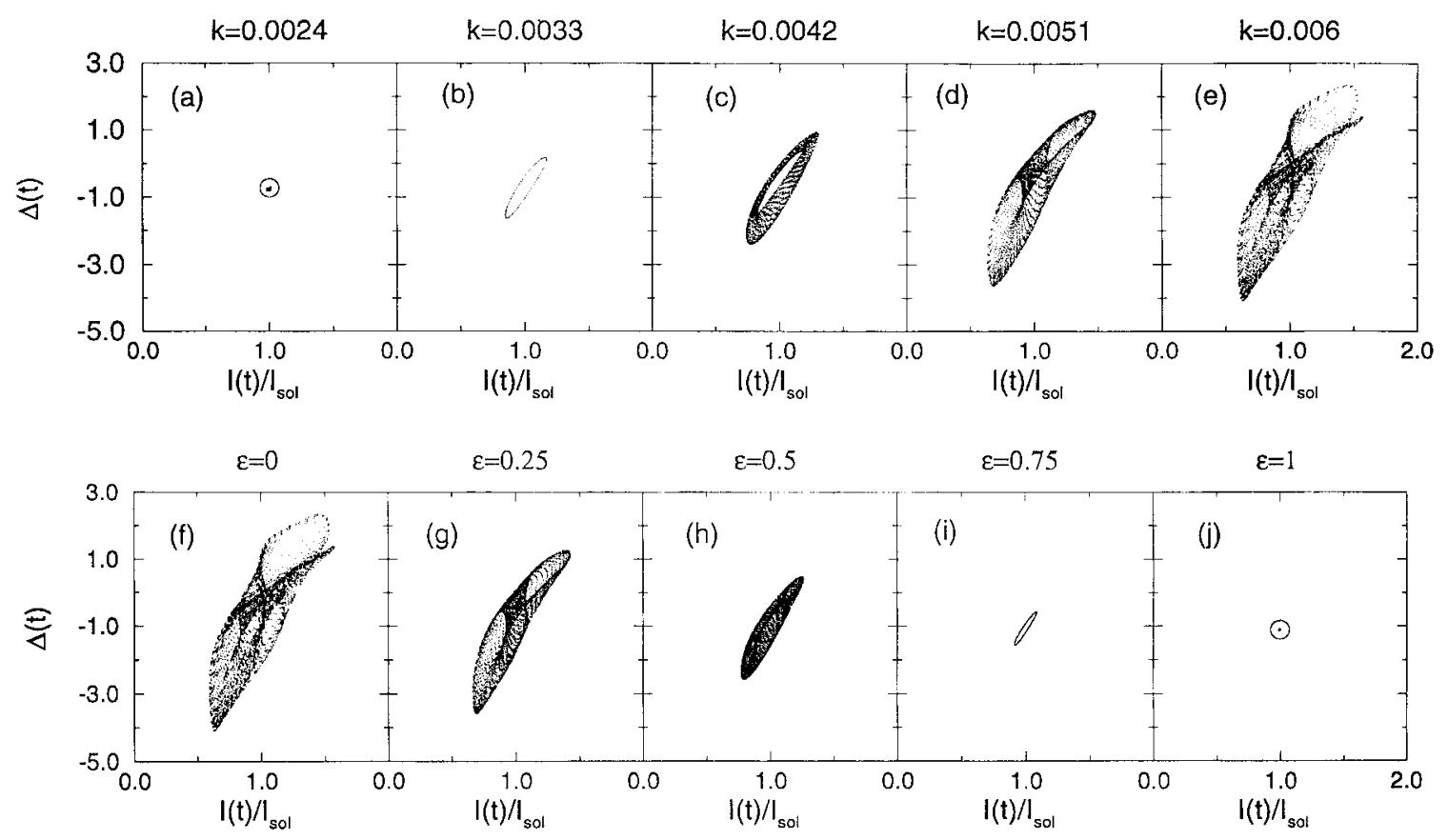

Fig. 1. Trajectories obtained integrating (5) and (3) of part I [11], with initial conditions chosen in mode A. In the first row, a linear gain is considered and the feedback is increased, while in the second row the feedback level is kept fixed and the gain saturation coefficient is increased ( $\varepsilon$ is measured in units of $7.5 \times 10^{-24} \mathrm{~m}^{3}$ ). The circles indicate the position of the fixed point corresponding to the perturbed laser mode.

analytical expression for the feedback above which a given mode becomes undamped is derived.

\section{RESULTS}

In this section, we present the results of the numerical integration of the Lang and Kobayashi equations

$$
\begin{aligned}
\frac{d\left[E(t) e^{i \phi(t)}\right.}{d t}= & \left(\frac{1+i \alpha}{2}\right)\left(G-\frac{1}{\tau_{p}}\right) E(t) e^{i \phi(t)} \\
& +\frac{k}{\tau_{\text {in }}} E(t-\tau) e^{i \phi(t-\tau)} e^{-i \omega_{o} \tau} . \\
\frac{d N(t)}{d t}= & J-\frac{N(t)}{\tau_{s}}-G E(t)^{2} .
\end{aligned}
$$

In these equations, the field amplitude is normalized such that $V E(t)^{2}$ is the total photon number in the laser waveguide (where $V$ is the volume of the active region). $\tau$ is the delay time $(\tau=2 L / c$ where $L$ is the length of the optical path and $c$ is the velocity of light), $\tau_{s}$ is the carrier lifetime, $\tau_{p}$ is the photon lifetime, and $\tau_{\text {in }}$ is the round-trip time in the laser cavity. $k$ is the feedback parameter, i.e., $k^{2}$ is the power reflected from the external cavity relative to the power reflected from the laser mirror. $\alpha$ is the linewidth enhancement factor and $J$ is the current density in carriers per unit volume and unit time. In the numerical simulation, the values used for these parameters are $\alpha=6, G=G_{N}\left(N-N_{o}\right)\left(1-E^{2}\right)$ with $G_{N}=1.1 \times 10^{-12} \mathrm{~m}^{3} / \mathrm{s}$, and $N_{o}=1.1 \times 10^{24} \mathrm{~m}^{-3} \cdot \tau_{s}=$ $2 \mathrm{~ns}, \tau_{p}=2 \mathrm{ps}, \tau_{\mathrm{in}}=8 \mathrm{ps}, \tau=2 \mathrm{~ns}, \omega_{o} \tau=\pi / 2$, and $J=$ $2 J_{\text {th }}\left(J_{\text {th }}\right.$ being the threshold current density).

The initial conditions are chosen in the external cavity modes of the laser, which are the fixed points of (3) and (4) and written in the form $E^{2}(t)=I_{s}, \phi(t)=\left(\omega_{s}-\omega_{o}\right) t, N(t)=N_{s}$ to satisfy

$$
\begin{aligned}
& \omega_{s} \tau-\omega_{o} \tau=-\left(k / \tau_{\text {in }}\right) \tau\left[\alpha \cos \left(\omega_{s} \tau\right)+\sin \left(\omega_{s} \tau\right)\right] \\
& G_{N}\left(N_{s}-N_{o}\right)\left(1-\varepsilon I_{s}\right)=1 / \tau_{p}-2\left(k / \tau_{\text {in }}\right) \cos \left(\omega_{s} \tau\right) \\
& J-N_{s} / \tau_{s}-G_{N}\left(N_{s}-N_{o}\right)\left(1-\varepsilon I_{s}\right) I_{s}=0
\end{aligned}
$$

The evolution of two particular modes are studied in detail: the perturbed laser mode (mode $\mathrm{A}$, which has $\omega_{s} \tau \approx-1$ ) and the first compound cavity mode (mode $\mathrm{B}$, which has $\omega_{s} \tau \approx 4.5$ ). The feedback level $k$ and the gain saturation coefficient $\varepsilon$ are the free parameters of our study (as in part I, $\varepsilon$ is measured in units of $7.5 \times 10^{-24} \mathrm{~m}^{3}$ ).

\section{A. Behavior of Mode A}

We begin by showing the behavior of the attractor originated from mode A when $k$ and $\varepsilon$ vary. In Fig. 1, the attractor is projected onto the plane formed by the normalized intensity $I(t) / I_{\mathrm{sol}}\left(I_{\mathrm{sol}}\right.$ being the intensity of the solitary laser) and the phase difference $\Delta=\omega_{0} \tau+\phi(t)-\phi(t-\tau)(\Delta / \tau$ being the average optical frequency, $\omega_{0}$ the threshold frequency of the solitary laser, $\tau$ the round-trip time in the external cavity, and $\phi$ the phase deviation from the phase of the stationary solitary laser).

For increasing feedback level and a fixed value of the parameter $\varepsilon$ (first row of Fig. 1), the stable mode [Fig. 1(a)] becomes a limit cycle [Fig. 1(b)] after a first Hopf bifurcation, and the limit cycle becomes a torus [Figs. 1(c) and (d)], after a second Hopf bifurcation. For $k=0.006$ [Fig. 1(e)], the torus period doubled, as will be shown below. This torus survives only for a short feedback interval $(k<0.0066)$ before it loses stability and gives rise to chaos.

For increasing $\varepsilon$ and fixed feedback level (second row of Fig. 1), the period-doubled torus reverses the route and 


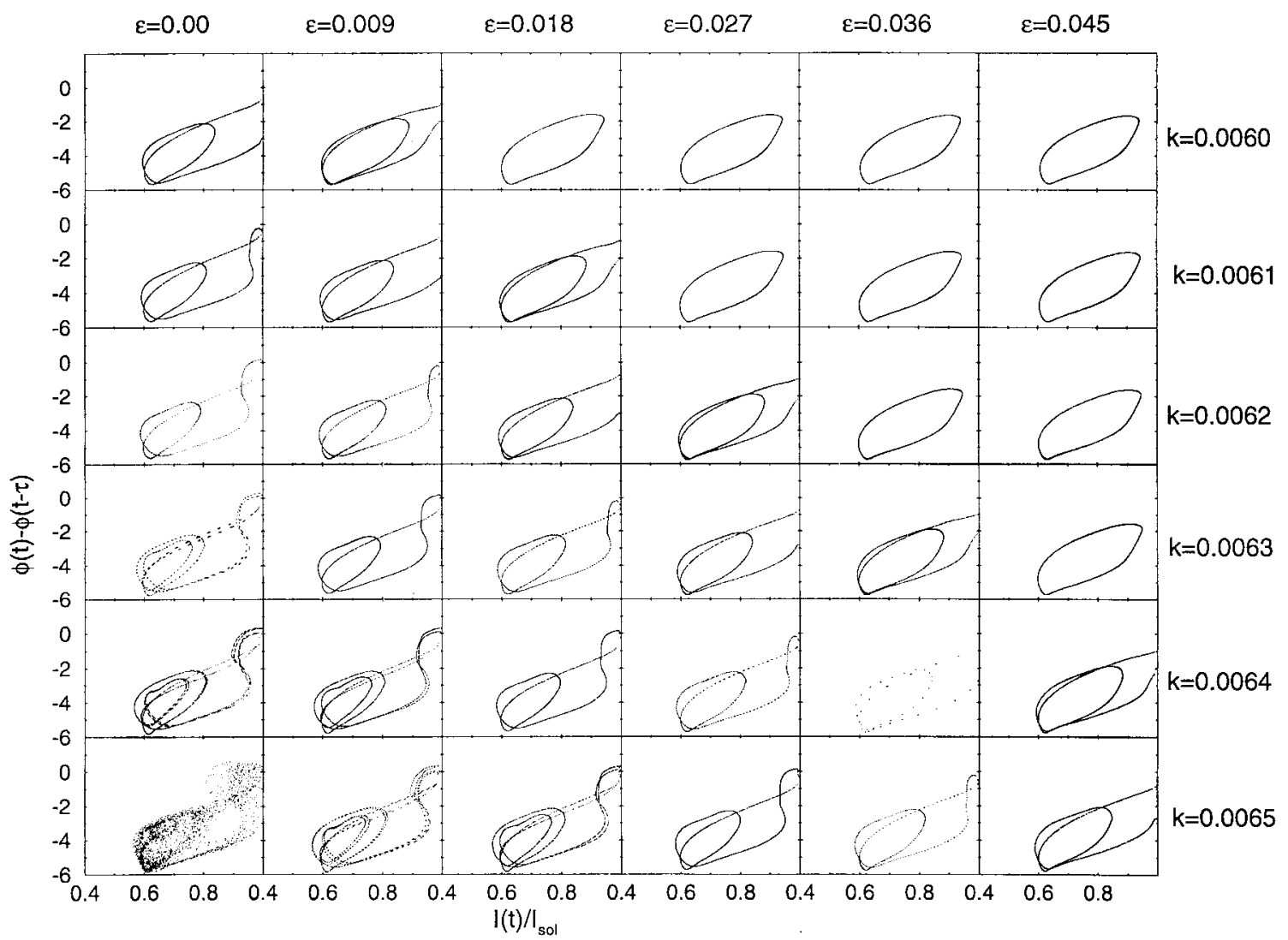

Fig. 2. Poincaré sections of attractor A for $0.006 \leq k \leq 0.0065$ and $0 \leq \varepsilon \leq 0.045$.

becomes a simple torus [Figs. 1(g) and (h)], then a limit cycle [Fig. 1(i)], and finally a stable mode again [Fig. 1(j)].

To gain insight into the deep features of the dynamics, we use the Poincaré section technique [12] and calculate the transversal cut of the attractor with the plane $N(t)=N_{\text {th }}\left(N_{\text {th }}\right.$ being the threshold carrier density). The intersection points are plotted in the plane formed by the normalized intensity $I(t) / I_{\mathrm{sol}}$ and phase delay $\phi(t)-\phi(t-\tau)$. In Fig. 2, the results obtained are presented in a matrix; the columns correspond to Poincaré sections that have equal (and different) $k$ while the rows correspond to Poincaré sections that have equal $k$ and different $\varepsilon$. The feedback was varied in the range $0.006 \leq k \leq 0.0065$, and the parameter $\varepsilon$ in the range $0 \leq \varepsilon \leq 0.045$ (the transversal cut of attractor $\mathrm{A}$ is also shown in Fig. 3 of part I, but in a larger parameter region).

The Poincare section shown in the upper left corner of Fig. 2 $(k=0.006, \varepsilon=0)$ is the transverse cut of the attractor shown in Fig. 1(e) with the plane $N=N_{\text {th }}$ and indicates that attractor $\mathrm{A}$ is a torus that period doubled at a lower feedback level.

Notice that the effect of increasing the feedback is opposite to the effect of increasing. In the former case, the attractor undergoes a few period-doubling bifurcations, while in the latter case the attractor undergoes a few inverse perioddoubling bifurcations. For example, the period-doubled torus located in the upper left corner of Fig. $2(k=0.006, \varepsilon=0)$ becomes a complicated attractor in the lower left corner $(k=$ $0.0065, \varepsilon=0)$. This attractor reverses the route as increases, and in the lower right corner $(k=0.0065, \varepsilon=0.045)$ is a torus that is almost equal to the torus located in the upper left corner.

Also, notice that the Poincaré sections for values of $(k, \varepsilon)$ that satisfy $k=k_{o}+n \Delta k, \varepsilon=\varepsilon_{o}+n \Delta \varepsilon$ with $\Delta k \approx$ $0.0001, \Delta \varepsilon \approx 0.009$, and $n$ integer (i.e., the ones that are located in the diagonals that go from up-left to down-right) are all approximately equal. This result suggests that, if the parameters $(k, \varepsilon)$ are varied certain amounts $(\Delta k, \Delta \varepsilon)$ with $\Delta k / \Delta \varepsilon \approx 0.01$, the dynamics of the attractor remains approximately unchanged.

Attractor A loses stability when the feedback level is increased above a certain critical value, but it recovers its stability if the value of the parameter $\varepsilon$ is slightly increased. In Fig. 3 of part I, we can see that for $\varepsilon=0$ attractor A loses stability for feedback levels above 0.0066 , but it is stable again if $\varepsilon$ is increased above 0.02 .

The critical feedback level $k_{c}$ above which attractor A loses stability augments with the value of the parameter $\varepsilon$. In Fig. 3 of part I, notice that for $\varepsilon=0$ attractor $\mathrm{A}$ is unstable for feedback levels above 0.0066, while for $\varepsilon=0.06$, it is unstable for feedback levels above 0.0072. In the parameter region shown in Fig. 3 of part I, when attractor A loses stability, chaos arises since the trajectory starts switching between the unstable attractors $\mathrm{A}$ and $\mathrm{B}$. As will be discussed below, for higher values of $\varepsilon$, when attractor A loses stability attractor B is still stable, and the trajectory evolves toward it.

The feedback level above which mode A becomes unstable and a limit cycle appears $\left(k_{l c}\right)$, and the feedback level above 


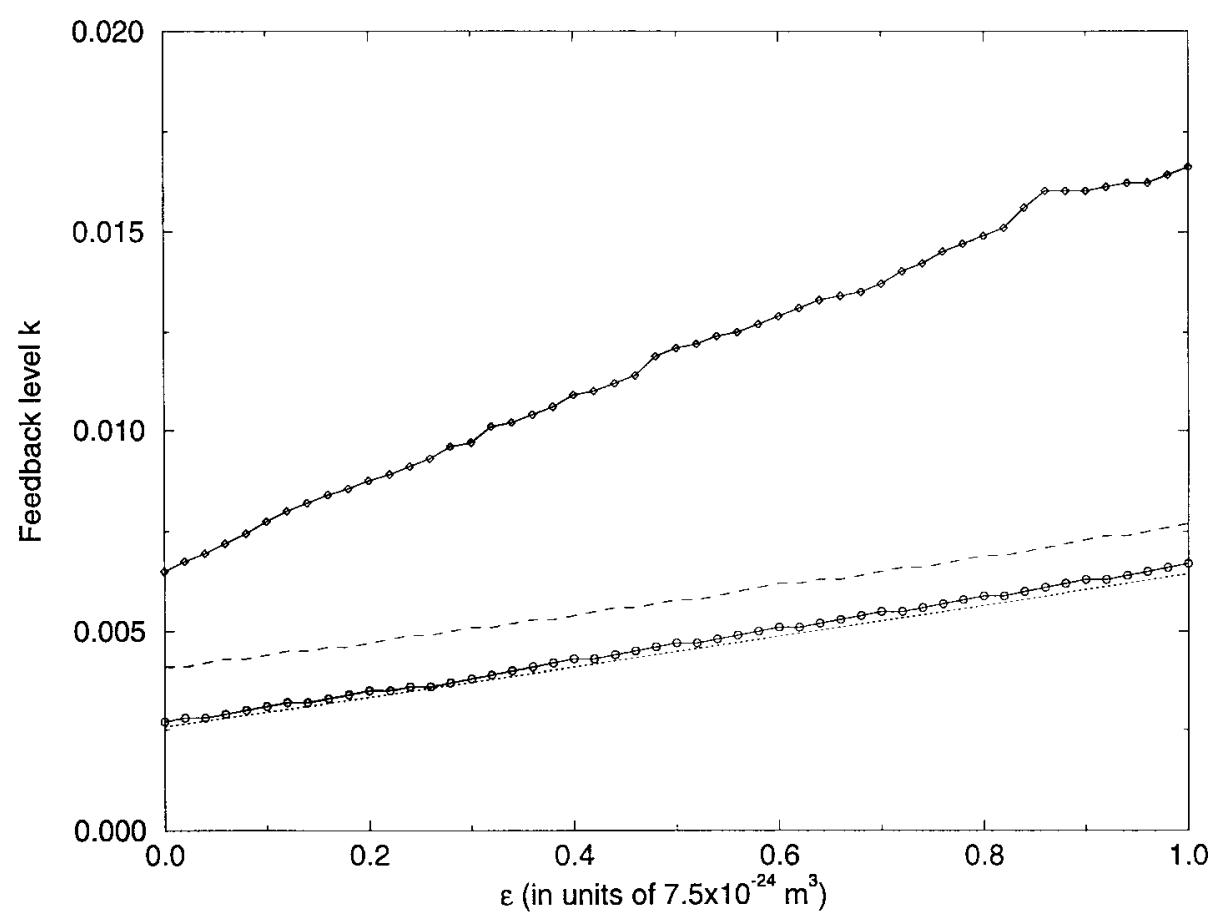

Fig. 3. Dependencies with the parameter of the feedback level above which attractor A becomes unstable $k_{c}$ (with $\diamond$ ), of the feedback level above which a limit cycle appears (calculated from the numerical simulation of the rate equations) $k_{l c}$ (with $\circ$ ), and of the feedback level above which a torus appears $k_{t}$ (dashed line). The dotted line represents the value of $k_{l c}$ obtained from the analytical expression (8).

which the limit cycle becomes unstable and a torus appears $\left(k_{t}\right)$, also increase with the value of the parameter $\varepsilon$. In Fig. 3, we have plotted the values of $k_{c}$ (with $\diamond$ ), $k_{l c}$ (with o), and $k_{t}$ (dashed line) calculated from the numerical simulation of the rate equations, as a function of the parameter $\varepsilon$. Notice that they are almost linear functions of $\varepsilon$ with positive slope, which means that the nonlinear gain increases the feedback level required to destabilize a fixed point, a limit cycle, or a chaotic attractor.

In Fig. 3, the dotted line represents the value of $k_{l c}$ calculated from the analytical expression (A14) derived in the Appendix:

$$
\kappa_{l c}=\frac{-\tau_{\mathrm{in}}\left(1 / \tau_{s}+G_{N} I_{\mathrm{sol}}+I_{\mathrm{sol}} \varepsilon / \tau_{p}\right)}{2 \sqrt{1+\alpha^{2}} \cos \left(\omega_{s} \tau-\arctan (\alpha)\right)} .
$$

Notice that (8) gives a very good approximation of the value of $k_{l c}$ obtained from the numerical simulation, but the latter is slightly higher than the value obtained from (8). Equation (8) demonstrates that the parameter renders a steady state more stable by increasing the feedback level above which the relaxation oscillations become undamped.

\section{B. Behavior of Mode B}

In Figs. 4 and 5, we can see that the effects of the parameters $k$ and $\varepsilon$ on attractor B are similar to those on attractor A. The effect of increasing the feedback while keeping the value of the parameter $\varepsilon$ fixed is opposite to the effect of increasing $\varepsilon$ while keeping the feedback fixed. In the first case, the stable mode [Fig. 4(a)] evolves into a limit cycle (Fig. 4(b) and (c)] and a torus [Fig. 4(d)], and the torus becomes a chaotic attractor [Fig. 4(e)]. In the second case, the chaotic attractor becomes a simple torus, which reverses the route and evolves into a limit cycle [Fig. 4(g)-(i)] and into a stable mode again [Fig. 4(j)].

The Poincaré section shown in the lower left corner of Fig. $5(k=0.0053, \varepsilon=0)$ is the transverse cut of the attractor shown in Fig. 4(e) with the plane $N=N_{\text {th }}$ and evidences that the attractor of Fig. 4(e) underwent several period-doubling bifurcations. Also, notice in Fig. 5 that if the parameters $(k, \varepsilon)$ are varied certain amounts $(\Delta k, \Delta \varepsilon)$, with $\Delta k \approx 0.0001 n, \Delta \varepsilon \approx 0.009 n$, and $n$ integer, the Poincaré section of attractor B remains approximately unchanged (i.e., the Poincaré sections that are located in the diagonals that go from up-left to down-right are approximately equal).

Attractor B loses stability if the feedback is increased above a certain critical value, but if $\varepsilon$ is slightly increased, the attractor becomes stable again. As an example, notice in Fig. 3 of part I that for $(k=0.0062, \varepsilon=0.02)$ only attractor $\mathrm{A}$ is stable, but for $(k=0.0062, \varepsilon=0.04)$ attractors $\mathrm{A}$ and $\mathrm{B}$ are stable.

Fig. 6 shows how $k_{c}, k_{l c}$ and $k_{t}$ of attractor $\mathrm{B}$ depend on the parameter $\varepsilon$. The results obtained for attractor B differ from those obtained for attractor A essentially by the fact that mode $\mathrm{B}$ has two associated attractors, which are both originated from mode B but at different feedback levels, as will be discussed below.

In Fig. 6, the (o) indicates the value of $k_{l c}$ above which a limit cycle arises from the stable mode B, calculated by direct numerical integration. This value is in excellent agreement with the value predicted by (8) (dotted line). The dashed line indicates the feedback level above which the limit cycle becomes a torus, and the $(\diamond)$ indicates the feedback level above which the chaotic attractor originated from the torus loses stability. 

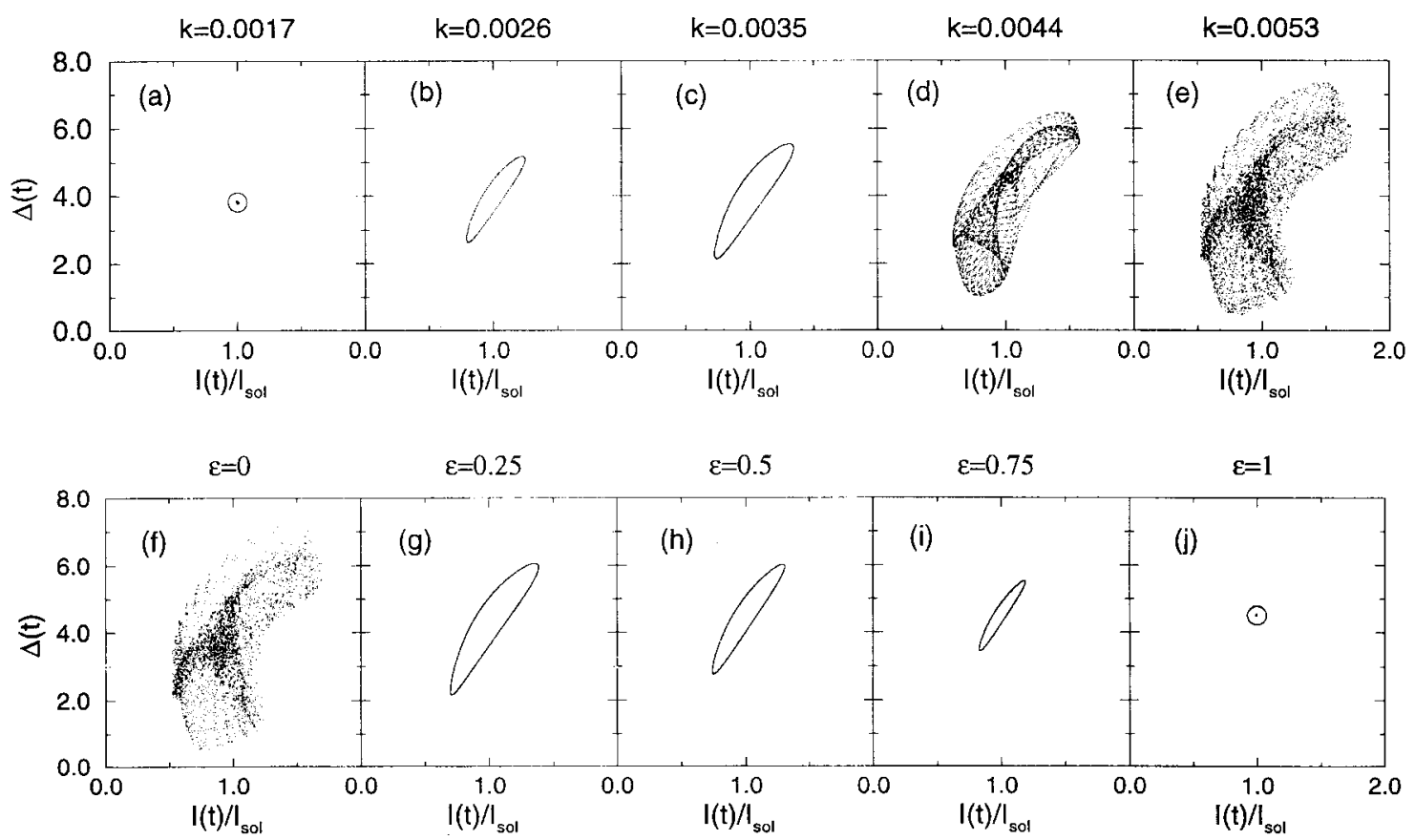

Fig. 4. Phase portraits of the attractor originated from mode B for increasing feedback level and for increasing $\varepsilon$. The circles indicate the position of the fixed point corresponding to mode B.

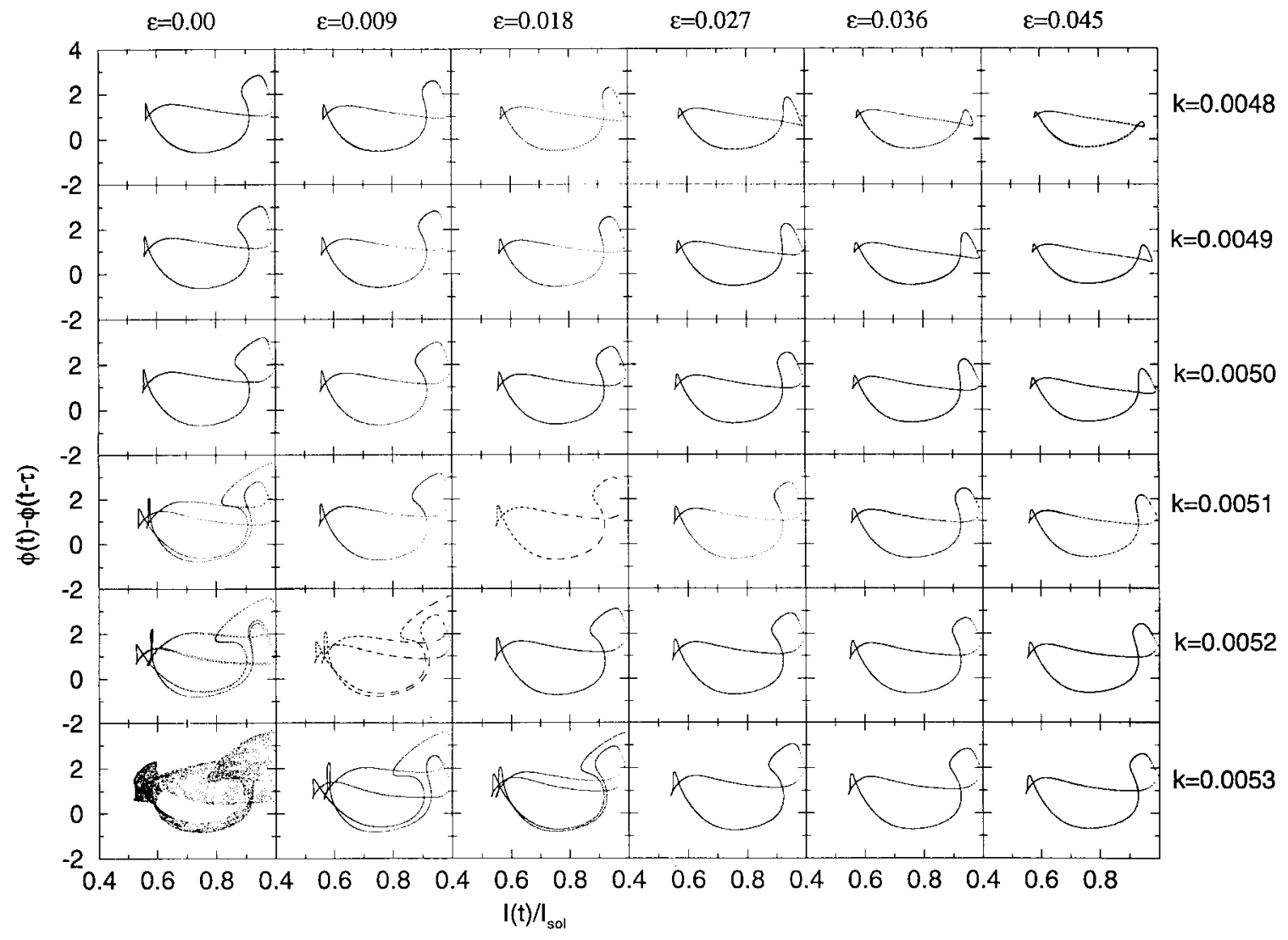

Fig. 5. Poincaré sections of attractor $\mathrm{B}$ for $0.0048 \leq k \leq 0.0053$ and $0 \leq \varepsilon \leq 0.045$.

For $\varepsilon=0$ and feedback levels slightly above $k=0.0053$, attractor B loses stability and the trajectory jumps to attractor A. Attractor A survives for a short feedback interval, and for feedback levels above 0.0066 the trajectory switches randomly between the unstable attractors $\mathrm{A}$ and $\mathrm{B}$ (see, e.g., Fig. 3 of part I). However, at a somewhat larger feedback $(k \approx$ 0.0083 ), a new limit cycle is created, which oscillates around the position of the unstable mode B. Our numerical results on 


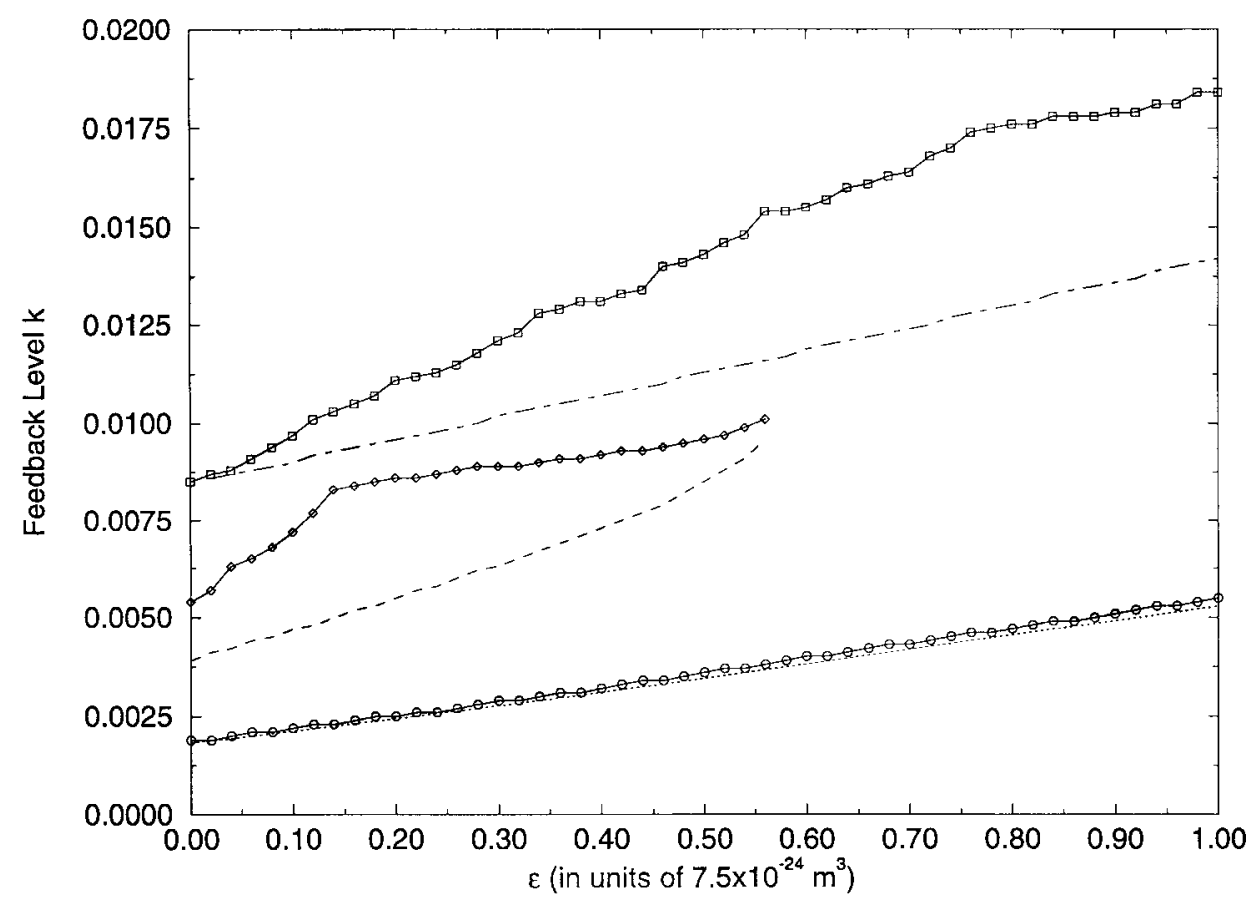

Fig. 6. Dependencies with the parameter $\varepsilon$ of $k_{c}$ (with $\left.\diamond\right), k_{l c}$ (with o), and $k_{t}$ (with dashed line) for attractor B. The dotted line shows the value of $k_{l c}$ predicted by (8). A second limit cycle is created from the unstable mode B, and the dashed-dotted line indicates the feedback level above which a torus emerges from this limit cycle, and the $(\square)$ indicates the feedback level above which the attractor originated from this torus loses stability.

the birth of this limit cycle are consistent with previous studies [5], [14] and suggest that the limit cycle is originated from an unstable limit cycle created from the unstable mode B, which bifurcates into a stable limit cycle and an unstable torus.

For $\varepsilon=0$, the second limit cycle originated from mode $\mathrm{B}$ exists for only a small $k$ interval, and for feedback levels above $k=0.0085$ the trajectory switches between attractors A and $B$ again. However, an increase of the value of the parameter $\varepsilon$ increases the feedback range where this new limit cycle exists. Even more, for higher values of the parameter $\varepsilon$, as the feedback increases the limit cycle follows the usual quasiperiodic route and turns into a torus which evolves into a chaotic attractor before losing stability. Therefore, in Fig. 6, the dashed-dotted line indicates the feedback level above which a torus emerges from the second limit cycle $\left(k_{t, 2}\right)$, and the $(\square)$ indicates the feedback level above which the attractor originated from this torus loses stability $\left(k_{c, 2}\right)$.

Notice that $k_{t, 2}$ and $k_{c, 2}$ are increasing functions of $\varepsilon$, and that the feedback range where the second attractor $\mathrm{B}$ exist also increases with the value of the parameter $\varepsilon$. However, the feedback range where the "old" attractor B exists increases with $\varepsilon$ for values of $\varepsilon$ below 0.16 , but for values of $\varepsilon$ above 0.16 , it diminishes until $\varepsilon \approx 0.58$, where it disappears. The annihilation of the old attractor B seems to be caused by the apparition of the new attractor B, since both attractors coexist for a short feedback interval, but the new attractor is strongly damped and eventually causes the trajectory to switch to it.

It is interesting to compare the previous results with those of our early work [15], where a linear gain was considered. For low values of $\varepsilon(\varepsilon<0.16)$ the route to chaos found here is essentially the same one that was found in [15], i.e., a period-doubling route of a 2-D torus. The main effect of gain saturation is to increase the feedback level above which chaos arises.

For $\varepsilon>0.16$, when attractor A loses stability attractor B is still stable and therefore the trajectory evolves toward it. Thus, for high values of not only the transition $\mathrm{A} \rightarrow \mathrm{B}$ instead of $\mathrm{A} \rightarrow$ chaos occurs, but also the attractors have a more complicated and higher dimensional structure. The route to chaos becomes more complex, and in the attractors the apparition of a third frequency or a high-order frequency locking are precursors of chaos. Nevertheless, for high values of $\varepsilon$, the effects of the parameters $k$ and $\varepsilon$ are still opposite: an increase of $k$ causes an attractor to become chaotic and eventually unstable, while an increase of $\varepsilon$ causes the attractor to recover its stability and reverse the route. For example, Figs. 7 and 8 show the effects of the parameters $k$ and $\varepsilon$ in attractors A and B for $\varepsilon=0.4$. Notice that according to Fig. 6, the transition to chaos for $\varepsilon=0.4$ occurs, for attractor $\mathrm{B}$, at $k=0.0131$, and Fig. 8 shows that attractor B is already chaotic for $k=0.0123$. However, let us remark that the transition to chaos occurs before the attractor loses stability. The value of $k_{c}$ plotted in Fig. 6 indicates not the critical feedback level above which attractor B becomes chaotic (this value is difficult to determine numerically precisely, since the transition to chaos is a smooth transition), but the value of $k$ above which attractor B loses stability (since the trajectory switches to another attractor, $k_{c}$ is a value that is easier to determine numerically).

Finally, let us revisit the results of [13], where the experimentally measured visibility curves of a laser operating in the coherence collapse state were numerically reproduced by calculating the field autocorrelation function with the Lang and Kobayashi model (the equations employed in [13] were 


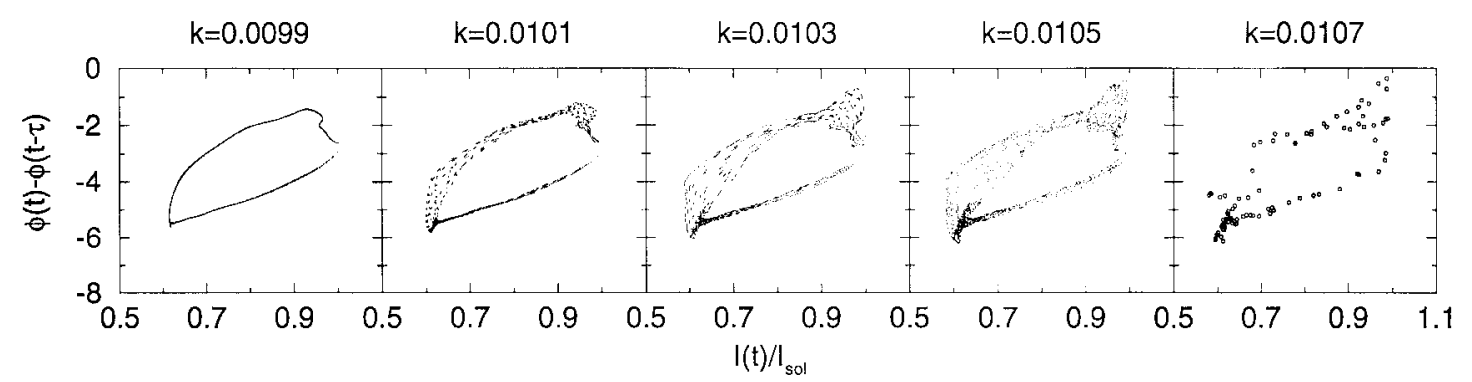

(a)

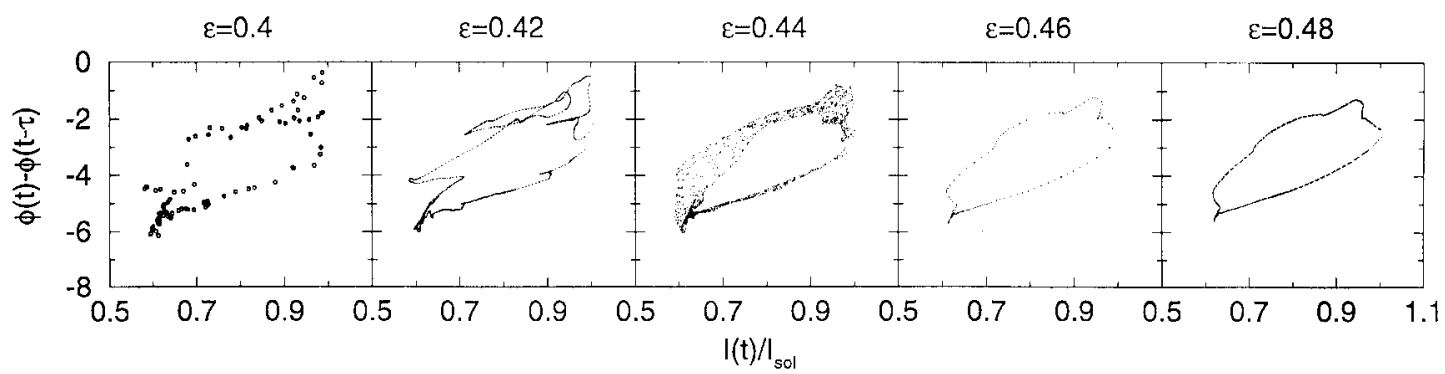

(b)

Fig. 7. Effect of the parameters $k$ and $\varepsilon$ on the Poincaré section of attractor A. For $\varepsilon=0.4$ and increasing feedback, the torus undergoes a third Hopf bifurcation and becomes a three-dimensional torus. For values of $k$ above 0.0107, the attractor loses stability after a high-order frequency locking (the points of the Poincaré section are indicated with small circles). By increasing the parameter $\varepsilon$, the attractor reverses the route and after an inverse Hopf bifurcations becomes a simple torus again.

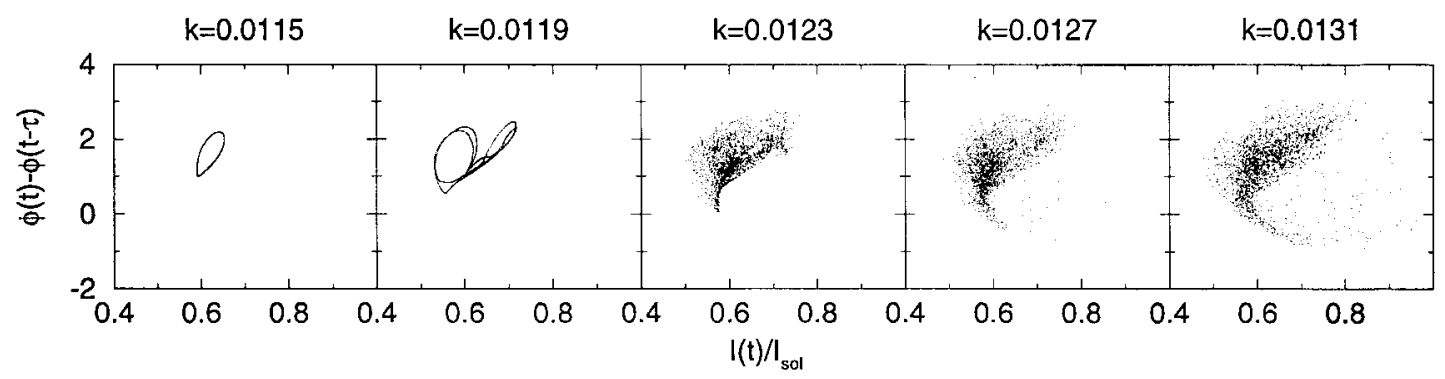

(a)

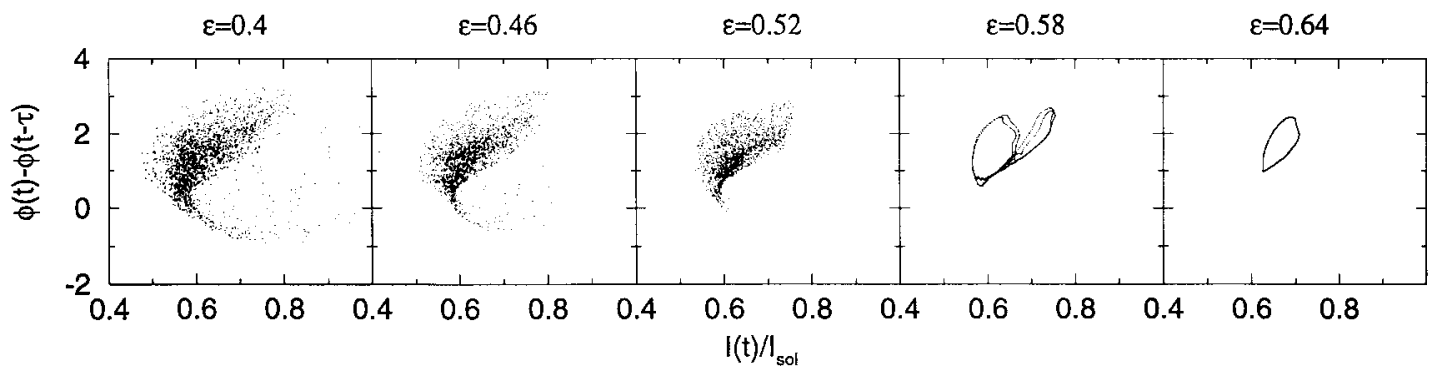

(b)

Fig. 8. Effect of the parameters $k$ and $\varepsilon$ on the Poincaré section of the "second" attractor B. For $\varepsilon=0.4$ and increasing feedback, the torus undergoes first a period-doubling bifurcation and then a third Hopf bifurcation and becomes a 3-D torus. Further increase of $k$ causes the torus to become a chaotic attractor before losing stability. For increasing $\varepsilon$, the route is reversed in an identical manner, i.e., the chaotic attractor turns to into a 3-D torus, then a period-doubled torus, and finally a simple torus.

(5) and (3) of part I). In the experiments, the amount of light fed back into the laser cavity was controlled with a variable attenuator and the visibility was measured for four different attenuations of the light (the attenuation was measured in arbitrary units as $0.7,0.8,0.9$, and 1.0 ; the value $0.7(1.0)$ corresponding to the lower (higher) feedback level). Even though the theoretical analysis revealed that there was a best value of $\varepsilon$ to fit the experimental measurements, good fits were also found using different values of $\varepsilon$, the higher the value of $\varepsilon$ employed, the higher the values of $\gamma_{1}, \gamma_{2}, \gamma_{3}$, and $\gamma_{4}$ that fitted the measurements ( $\gamma$ in [13] corresponds to $k / \tau_{\text {in }}$ in this paper). These values are plotted as a function of $\varepsilon$ in 


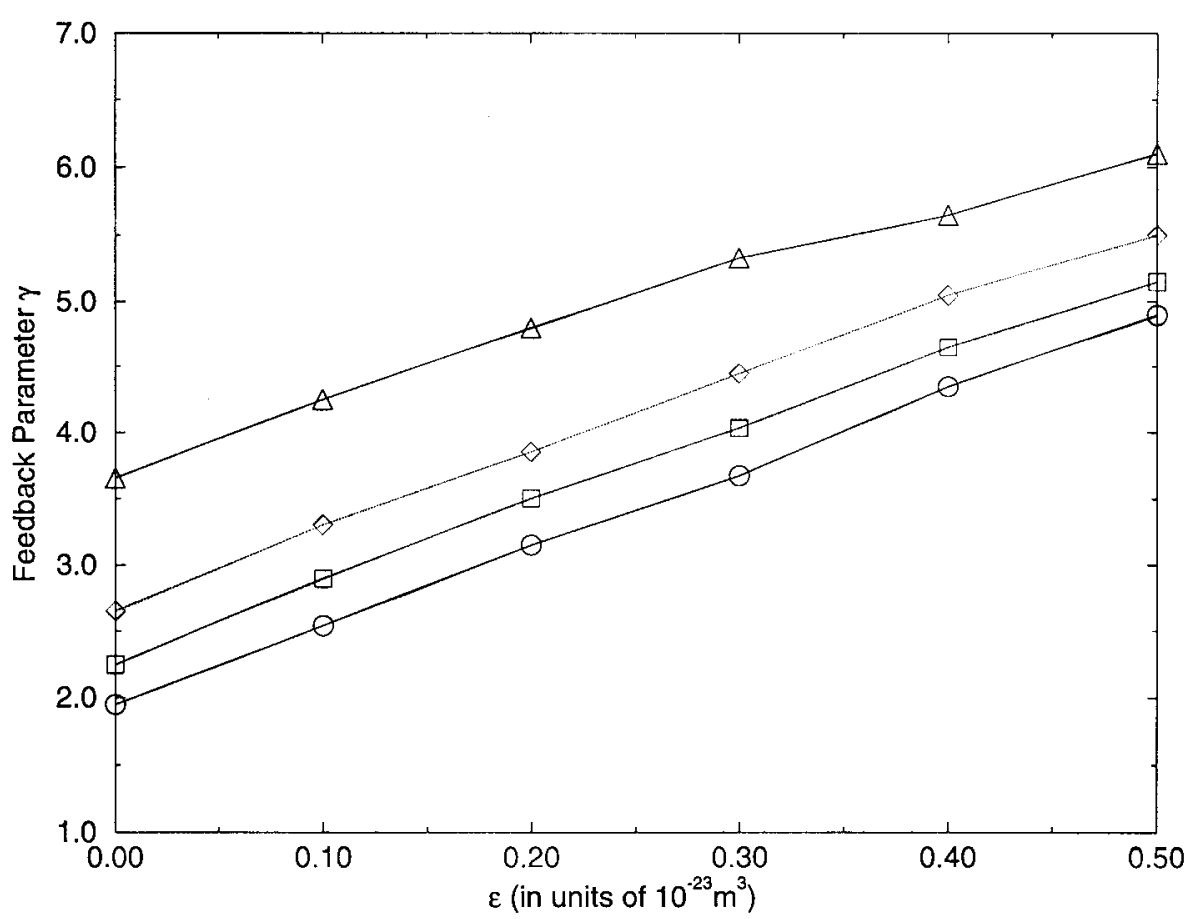

Fig. 9. Feedback levels employed in [13] to reproduce the visibility curves, as a function of the parameter $\varepsilon$ ( $\gamma$ in [13] corresponds to $k / \tau_{\text {in }}$ in this paper). $\gamma_{1}$ (the feedback level that fits the curve with higher attenuation) is indicated with a circle, $\gamma_{2}$ is indicated with a square, $\gamma_{3}$ is indicated with a diamond, and $\gamma_{4}$ (the feedback level that fits the curve with lower attenuation) is indicated with a triangle.

Fig. 9. Clearly, the plot shows four linear relations with the same positive slope. This suggests that the visibility curve of the laser remains approximately unchanged if the parameters $(k, \varepsilon)$ are varied accordingly to this linear relation. This result again emphasizes that the effects of the parameters $k$ and $\varepsilon$ are opposite, even in the coherence collapse state.

\section{CONCLUSION}

We have presented a detailed comparison of the effects of weak optical feedback and nonlinear gain in the dynamics of a single-mode semiconductor laser. The main conclusions of our study are as follows.

First, we have found that even though the parameters $k$ and $\varepsilon$ play very different roles in the Lang and Kobayashi model since they appear in terms that represent independent physical contributions to the rate of variation of the complex electric field, they effect is surprisingly opposite in all the parameter regions studied. We have found that the route to chaos that the attractors follow for increasing $k$ and a fixed value of $\varepsilon$ is reversed in an almost identical manner for increasing $\varepsilon$ and a fixed value of $k$. Also, we have shown that the Poincaré section of an attractor remains approximately unchanged if the values of the parameters $(k, \varepsilon)$ are both increased or decreased certain amounts $(\Delta k, \Delta \varepsilon)$. The variations $(\Delta k, \Delta \varepsilon)$ that leave a Poincare section unchanged depend on the attractor, but in all cases studied they satisfy $\Delta k \Delta \varepsilon>0$, i.e., an increase of the feedback $\Delta k$ can be compensated by an increase of the parameter $\varepsilon$ in $\Delta \varepsilon$. In addition, we have shown that the values of $k$ and $\varepsilon$ that were used in [13] to fit four experimentally measured visibility curves of a laser operating in the coherence collapse regime satisfy four linear relations with the same positive slope. This suggests that the visibility of the laser remains approximately unchanged if the values of the parameters $(k, \varepsilon)$ are both increased or decreased according to the linear relation.

Second, we have found that the value of the parameter $\varepsilon$ increases the stability region of an attractor by pulling up the feedback level above which an attractor loses stability. The feedback level above which the relaxation oscillations of a given mode become undamped and a periodic limit cycle appears $\left(k_{l c}\right)$, the feedback level above which a quasi-periodic torus emerges from a limit cycle $\left(k_{t}\right)$, and the feedback level above which a chaotic attractor loses stability $\left(k_{c}\right)$ are all increasing functions of the parameter $\varepsilon$. The value of $k_{l c}$ and its dependency with the parameter $\varepsilon$ is accurately predicted by the analytical expression derived from a small-signal analysis of the rate equations.

A qualitative explanation of the dependencies of the laser behavior on the amount of optical feedback and variations of the gain saturation coefficient is that both an increase of the feedback term and a decrease of the gain saturation term contribute to increase the intensity of the light inside the laser cavity. Our results are consistent with those of [16], where the effect of strong feedback was shown to result in an additional complex gain term, the real part being related to the gain due to the optical feedback, and the imaginary part being related to a frequency shift.

The stabilizing effect of nonlinear gain and the destabilizing effect of optical feedback are well known and have been observed experimentally. However, the surprising and intriguing way in which both effects are opposite and compensate each other has not been reported previously to our knowledge. 
Even though the phenomenon is beyond the possibilities of experimental detection, since the parameter cannot be varied in the experiments, it is an interesting phenomenon that deserves further clarification.

\section{APPENDIX}

Since in the literature the previous stability analysis were based on (6) and (3) of part I [5], [17], in this appendix, we perform a small-signal analysis of the rate equations (3) and (4) near an initally stable mode $\left(I_{s}, \omega_{s}, N_{s}\right)$ and derive an analytical expression for the feedback level $k_{l c}$ above which the mode loses stability and a limit cycle appears.

To first-order, the deviation $(\delta I, \delta \phi, \delta N)$ from the stationary solution satisfies the equations

$$
\begin{aligned}
\frac{d(\delta I)}{d t}= & I_{s} G_{N}^{\prime} \delta N-G_{s} \varepsilon^{\prime} I_{s} \delta I-\gamma \cos \left(\omega_{s} \tau\right) \\
& \cdot[\delta I(t)-\delta I(t-\tau)] \\
& -2 \gamma I_{s} \sin \left(\omega_{s} \tau\right)[\delta \phi(t)-\delta \phi(t-\tau)] \\
\frac{d(\delta \phi)}{d t}= & \frac{1}{2} \alpha G_{N}^{\prime} \delta N-\frac{1}{2} \alpha G_{s} \varepsilon^{\prime} \delta I \\
& +\frac{\gamma}{2 I_{s}} \sin \left(\omega_{s} \tau\right)[\delta I(t)-\delta I(t-\tau)] \\
& -\gamma \cos \left(\omega_{s} \tau\right)[\delta \phi(t)-\delta \phi(t-\tau)] \\
\frac{d(\delta N)}{d t}= & -\left(1 / \tau_{s}+I_{s} G_{N}^{\prime}\right) \delta N+\left(I_{s} G_{s} \varepsilon^{\prime}-G_{s}\right) \delta I(t)
\end{aligned}
$$

where

$$
\begin{aligned}
\gamma & =k / \tau_{\mathrm{in}}, G_{N}^{\prime}=G_{N}\left(1-\varepsilon I_{s}\right), \varepsilon^{\prime}=\varepsilon /\left(1-\varepsilon I_{s}\right) \\
G_{s} & =G_{N}^{\prime}\left(N_{s}-N_{o}\right)=1 / \tau_{p}-2 \gamma \cos \left(\omega_{s} \tau\right)
\end{aligned}
$$

Since for typical parameter values $\varepsilon I_{s} \ll 1, G_{N}^{\prime} \approx G_{N}$ and $\varepsilon^{\prime} \approx \varepsilon$. In the following, we will replace $G_{N}^{\prime}$ by $G_{N}$ and $\varepsilon^{\prime}$ by $\varepsilon$. The determinant of the Laplace transform is

$$
\begin{aligned}
D(s)=s^{3} & +2\left[\gamma\left(1-e^{-s \tau}\right) \cos \left(\omega_{s} \tau\right)+\Gamma\right] s^{2} \\
+ & {\left[\gamma^{2}\left(1-e^{-s \tau}\right)^{2}+2 \Gamma_{1} \gamma\left(1-e^{-s \tau}\right)\right.} \\
& \cdot\left(\cos \left(\omega_{s} \tau\right)-\alpha \sin \left(\omega_{s} \tau\right)\right)+4 \Gamma_{N} \gamma\left(1-e^{-s \tau}\right) \\
& \left.\cdot \cos \left(\omega_{s} \tau\right)+\Omega_{R}^{2}\right] s \\
+ & 2 \Gamma_{N} \gamma^{2}\left(1-e^{-s \tau}\right)^{2}+\Omega_{R}^{2} \gamma\left(1-e^{0 s \tau}\right) \\
& \cdot\left(\cos \left(\omega_{s} \tau\right)-\alpha \sin \left(\omega_{s} \tau\right)\right)
\end{aligned}
$$

where

$$
\begin{aligned}
\Gamma_{N} & =1 / 2\left(1 / \tau_{s}+G_{N} I_{s}\right) \\
\Gamma_{I} & =1 / 2 G_{s} \varepsilon I_{s} \\
\Gamma & =\Gamma_{I}+\Gamma_{N} \\
\Omega_{R}^{2} & =G_{s} I_{s} G_{N}\left[1+\varepsilon /\left(G_{N} \tau_{s}\right)\right] .
\end{aligned}
$$

As was discussed in part I, the stationary intensity $I_{s}$ satisfies [11, eq. (A.5)]):

$$
I_{s}=\frac{I_{\mathrm{sol}} / \tau_{p}+2 \gamma \cos \left(\omega_{s} \tau\right) /\left(G_{N} \tau_{s}\right)}{1 / \tau_{p}-2 \gamma \cos \left(\omega_{s} \tau\right)}\left[1-\frac{\varepsilon}{G_{N} \tau_{s}}\right] .
$$

Therefore, substituting (A4) and (A7) in (A6), we obtain

$$
\Omega_{R}^{2}=\left[I_{\mathrm{sol}} / \tau_{p}+2 \gamma \cos \left(\omega_{s} \tau\right) /\left(G_{N} \tau_{s}\right)\right] G_{N}\left[1-O^{2}\left(\varepsilon / G_{N} \tau_{s}\right)\right]
$$

which is independent to first-order of the value of the parameter $\varepsilon$. Moreover, the effect of the feedback on the stationary intensity $I_{s}$ can be neglected compared to the value of the stationary intensity of the solitary laser $I_{\text {sol }}$, and therefore $\Omega_{R}$ can be approximated by

$$
\Omega_{R}^{2} \approx G_{n} I_{\mathrm{sol}} / \tau_{p}=\omega_{R}^{2}
$$

where $\omega_{R}$ is the relaxation oscillation frequency of the solitary laser.

$\Gamma$ is the decay rate of the relaxation oscillations and is given by

$$
\begin{aligned}
\Gamma & =\frac{1}{2}\left(\frac{1}{\tau_{s}}+G_{N} I_{s}+G_{s} \varepsilon I_{s}\right) \\
& \approx \frac{1}{2}\left\{\frac{1}{\tau_{s}}+G_{N} I_{\mathrm{sol}}+\frac{I_{\mathrm{sol}}}{\tau_{p}} \varepsilon\right\}=\Gamma_{R}
\end{aligned}
$$

where $\Gamma_{R}$ is the decay rate of the solitary laser (in (A10) the effect of the feedback on the stationary intensity was also neglected).

Assuming $\omega_{R}^{2} \gg \gamma^{2}, \omega_{R}^{2} \gg \gamma \Gamma_{1}$, and $\omega_{R}^{2} \gg \gamma \Gamma_{N}$, the system determinant can be approximated by

$$
\begin{aligned}
D(s)= & s^{3}+2\left[\gamma\left(1-e^{-s \tau}\right) \cos \left(\omega_{s} \tau\right)+\Gamma_{R}\right] s^{2}+\omega_{R}^{2} s \\
& +\omega_{R}^{2}\left(\cos \left(\omega_{s} \tau\right)-\alpha \sin \left(\omega_{s} \tau\right)\right) \gamma\left(1-e^{-s \tau}\right)
\end{aligned}
$$

(notice that in (A11) the parameter appear only in the value of $\left.\Gamma_{R}\right)$.

A stable mode becomes unstable when a zero of $D(s)$ passes the imaginary axis. By inserting $s=j \Omega$ in (A11) and separating the real and imaginary parts, we obtain the following relations:

$$
\begin{aligned}
& -2\left[\gamma_{l c}(1-\cos (\Omega \tau)) \cos \left(\omega_{s} \tau\right)+\Gamma_{R}\right] \Omega^{2} \\
& +\omega_{R}^{2}\left(\cos \left(\omega_{s} \tau\right)-\alpha \sin \left(\omega_{s} \tau\right)\right) \gamma_{l c}(1-\cos (\Omega \tau))=0 \\
& \left.-\Omega^{3}-2 \gamma_{l c} \sin (\Omega \tau) \cos \left(\omega_{s} \tau\right) \Omega^{2}\right) \\
& +\omega_{R}^{2} \Omega+\omega_{R}^{2}\left(\cos \left(\omega_{s} \tau-\alpha \sin \left(\omega_{s} \tau\right)\right) \gamma_{l c} \sin (\Omega \tau)=0\right.
\end{aligned}
$$

The onset angular frequency $\Omega$ and the onset feedback level $k_{l c}$ can be found solving the coupled equations (A12) and (A13). In general, $\Omega$ will be close to $\omega_{R}$ [4], [5], [17], and if $\omega_{R} \tau=(2 n+1) \pi$ with $n$ integer, the feedback level above which the mode becomes unstable and a limit cycle appears is

$$
k_{l c}=\frac{-\tau_{\mathrm{in}} \Gamma_{R}}{\cos \left(\omega_{s} \tau\right)+\alpha \sin \left(\omega_{s} \tau\right)} .
$$

As disscused in [17], this is a lower limit of the actual value of $k_{l c}$, which depends on the value of $\omega_{R} \tau$. We show in Section II that for our parameter values (A14) provides a very good approximation of the feedback level above which a mode becomes undamped. 


\section{REFERENCES}

[1] G. P. Agrawal and N. K. Dutta, Long-Wavelength Semiconductor Lasers. New York: Van Nostrand Reinhold, 1986.

[2] K. Petermann, Laser Diode Modulation and Noise. Dordrecht, The Netherlands: Kluwer, 1988.

[3] R. Lang and K. Kobayashi, "External optical feedback effects on semiconductor laser properties," IEEE J. Quantum Electron., vol. QE-16, pp. 347-355, 1980.

[4] B. Tromborg, J. H. Osmundsen, and H. Olesen, "Stability analysis for a semiconductor laser in an external cavity," IEEE J. Quantum Electron., vol. QE-20, pp. 1023-1032, 1984.

[5] J. MØrk, B. Tromborg, and J. Mark, "Chaos in semiconductor lasers with optical feedback: Theory and experiment," IEEE J. Quantum Electron., vol. 28 , pp. 93-108, 1992.

[6] C. H. Henry, "Theory of the linewidth of semiconductor lasers," IEEE J. Quantum Electron., vol. QE-18, pp. 259-264, 1982.

[7] J. Sacher, D. Baums, P. Panknin, W. Elsässer, and E. O. Göbel, "Intensity instabilities of semiconductor lasers under current modulation, external light injection, and delayed feedback," Phys. Rev. A, vol. 45, pp. 1893-1905, 1992.

[8] A. T. Ryan and G. P. Agrawal, "Optical-feedback induced chaos and its control in multimode semiconductor lasers," IEEE J. Quantum Electron. vol. 30, pp. 668-678, 1994

[9] K. Wada, T. Umeda, and Y. Cho, "Linewidth enhancement factor dependence of the instability in semiconductor lasers with delayed feedback and its influence on the analyzes," Opt. Commun., vol. 109, pp. 93-100, 1994.
[10] C. Masoller, A. C. Sicardi Schifino, and C. Cabeza, "The nonlinear gain and the onset of chaos in a semiconductor laser with optical feedback," J. Chaos, Solitons Fractals, vol. 6, pp 347-356, 1995.

[11] C. Masoller, "Implications of how the linewidth enhancement factor is introduced on the Lang and Kobayashi model," this issue, pp. 796-803.

[12] H. G. Schuster, Deterministic Chaos, 2nd ed. Weinheim, Germany: VCH, 1988.

[13] C. Masoller, C. Cabeza, and A. C. Sicardi Schifino, "Study of the effect of the nonlinear gain in the visibility of a semiconductor laser with incoherent feedback in the coherence collapsed regime: theory and experiment," IEEE J. Quantum Electron., vol. 31, pp. 1022-1029, 1995.

[14] A. Ritter and H. Haug, "Theory of the bistable limit cycle behavior of laser diodes induced by weak optical feedback," IEEE J. Quantum Electron., vol. 29, pp. 1064-1070, 1993.

[15] C. Masoller, A. C. Sicardi Schiffino, and C. Cabeza, "Chaotic properties of the coherence collapsed state of laser diodes with optical feedback," Opt. Commun., vol. 100, pp. 331-339, 1993.

[16] H. Kakiuchida and J. Ohtsubo, "Characteristics of a semiconductor laser with external feedback," IEEE J. Quantum Electron., vol. 30, pp. 2087-2097, 1994.

[17] A. Ritter and H. Haug, "Theory of laser diodes with weak optical feedback. I. Small-signal analysis and side-mode spectra," J. Opt. Soc. Amer. B, vol. 10, pp. 130-144, 1993.

C. Masoller, photograph and biography not available at the time of publication. 